\title{
Period Doubling Renormalization for Area-Preserving Maps and Mild Computer Assistance in Contraction Mapping Principle
}

\author{
Denis G. Gaidashev \\ Department of Mathematics, \\ Uppsala University, Box 480, \\ 75106 Uppsala, Sweden, \\ gaidash@math.uu.se
}

November 10, 2018

\begin{abstract}
A universal period doubling cascade analogous to the famous FeigenbaumCoullet-Tresser period doubling has been observed in area-preserving maps of $\mathbb{R}^{2}$. Existence of the "universal" map with orbits of all binary periods has been proved via a renormalization approach in (Eckmann et al 1984) and (Gaidashev et al 2011). These proofs use "hard" computer assistance.

In this paper we attempt to reduce computer assistance in the argument, and present a mild computer aided proof of the analyticity and compactness of the renormalization operator in a neighborhood of a renormalization fixed point: that is a proof that does not use generalizations of interval arithmetics to functional spaces - but rather relies on interval arithmetics on real numbers only to estimate otherwise explicit expressions. The proof relies on several instance of the Contraction Mapping Principle, which is, again, verified via mild computer assistance.
\end{abstract}




\section{Introduction: Period doubling universality for area- preserving maps}

Following the pioneering discovery of the Feigenbaum-Coullet-Tresser period doubling universality in unimodal maps (Feigenbaum 1978, Feigenbaum 1979, Tresser and Coullet 1978) universality has been demonstrated to be a rather generic phenomenon in dynamics.

To prove universality one usually introduces a renormalization operator in a functional space, and demonstrates that this operator has a hyperbolic fixed point.

Such renormalization approach to universality has been very successful in one-dimensional dynamics, and has led to explanation of universality in unimodal maps (Epstein 1986, Epstein 1989, Sullivan 1992, Lyubich 1999), critical circle maps (de Faria 1992, de Faria 1999, Yampolsky 2002, Yampolsky 2003) and holomorphic maps with a Siegel disks (McMullen 1998, Yampolsky 2007, Gaidashev and Yampolsky 2007). Universality has been also abundantly observed in higher dimensions, in particular, in two and more dimensional dissipative systems (cf. (Collet et al 1980), (Sparrow 1982)), in area-preserving maps, both as the period doubling universality (Derrida and Pomeau 1980, Helleman 1980, Benettin et al 1980, Collet et al 1981, Eckmann et al 1982, Eckmann et al 1984, Gaidashev and Koch 2008, Gaidashev and Johnson 2009, Gaidashev and Johnson 2009, Gaidashev et al 2011), and as the universality associated with the break-up of invariant surfaces (Shenker and Kadanoff 1982, MacKay 1982, MacKay 1983, Mehr and Escande 1984), and in Hamiltonian flows (Escande and Doveil 1981, Abad et al 2000, Abad et al 1998, Koch 2002, Koch 2004, Koch 2008, Gaidashev and Koch 2004, Gaidashev 2005, Kocić 2005, Khanin et al 2007).

In this paper we will consider a period doubling universality for area-preserving maps of the plane - an analogue of Feigenbaum-Coullet-Tresser universality in higher dimensions.

An infinite period doubling cascade in families of area-preserving maps was observed by several authors in early 80's (Derrida and Pomeau 1980, Helleman 1980, Benettin et al 1980, Bountis 1981, Collet et al 1981). A typical period doubling scenario can be illustrated with the area-preserving Hénon family (cf. (Bountis 1981)) :

$$
H_{a}(x, y)=\left(-y+1-a x^{2}, x\right) .
$$

Maps in this family posses a fixed point $((-1+\sqrt{1+a}) / a,(-1+\sqrt{1+a}) / a)$ which is stable for $-1<a<3$. When $a_{1}=3$ this fixed point becomes unstable, at the same time an orbit of period two is born with $H_{a}\left(x_{ \pm}, x_{\mp}\right)=\left(x_{\mp}, x_{ \pm}\right)$, $x_{ \pm}=(1 \pm \sqrt{a-3}) / a$. This orbit, in turn, becomes unstable at $a_{2}=4$, giving birth to a period 4 stable orbit. Generally, there exists a sequence of parameter values $a_{k}$, at which the orbit of period $2^{k-1}$ turns unstable, while at the same time a stable orbit of period $2^{k}$ is born. The parameter values $a_{k}$ accumulate on some $a_{\infty}$. The crucial observation is that the accumulation rate

$$
\lim _{k \rightarrow \infty} \frac{a_{k}-a_{k-1}}{a_{k+1}-a_{k}}=8.721 \ldots
$$


is universal for a large class of families, not necessarily Hénon.

Furthermore, the $2^{k}$ periodic orbits scale asymptotically with two scaling parameters

$$
\lambda=-0.249 \ldots, \quad \mu=0.061 \ldots
$$

To explain how orbits scale with $\lambda$ and $\mu$ we will follow (Bountis 1981). Consider an interval $\left(a_{k}, a_{k+1}\right)$ of parameter values in a "typical" family $F_{a}$. For any value $\alpha \in\left(a_{k}, a_{k+1}\right)$ the map $F_{\alpha}$ posses a stable periodic orbit of period $2^{k}$. We fix some $\alpha_{k}$ within the interval $\left(a_{k}, a_{k+1}\right)$ in some consistent way; for instance, by requiring that the restriction of $F_{\alpha_{k}}^{2^{k}}$ to a neighborhood of a stable periodic point in the $2^{k}$-periodic orbit is conjugate via a diffeomorphism $H_{k}$ to a rotation with some fixed rotation number $r$. Let $p_{k}^{\prime}$ be some unstable periodic point in the $2^{k-1}$-periodic orbit, and let $p_{k}$ be the further (from $p_{k}^{\prime}$ ) of the two stable $2^{k}$-periodic points that bifurcated from $p_{k}^{\prime}$. Then,

$$
\frac{1}{\lambda}=-\lim _{k \rightarrow \infty} \frac{\left|p_{k}-p_{k}^{\prime}\right|}{\left|p_{k+1}-p_{k+1}^{\prime}\right|}, \quad \frac{\lambda}{\mu}=-\lim _{k \rightarrow \infty} \frac{\rho_{k}}{\rho_{k+1}},
$$

where $\rho_{k}$ is the ratio of the eigenvalues of $D H_{k}\left(p_{k}\right)$.

This universality can be explained rigorously if one shows that the renormalization operator

$$
R[F]=\Lambda_{F}^{-1} \circ F \circ F \circ \Lambda_{F},
$$

where $\Lambda_{F}$ is some $F$-dependent coordinate transformation, has a fixed point, and the derivative of this operator is hyperbolic at this fixed point.

It has been argued in (Collet et al 1981) that $\Lambda_{F}$ is a diagonal linear transformation. Furthermore, such $\Lambda_{F}$ has been used in (Eckmann et al 1982) and (Eckmann et al 1984) in a computer assisted proof of existence of the renormalization fixed point. This is the strongest result to date concerning the existence of the renormalization fixed point.

The problem has been further studied in (Gaidashev and Koch 2008), where the authors have demonstrated that the period doubling renormalization fixed point of (Eckmann et al 1984) is "almost" one dimensional, in the sense that it is close to the following Hénon-like map:

$$
H_{*}(x, u)=(\phi(x)-u, x-\phi(\phi(x)-u)),
$$

where $\phi$ solves

$$
\phi(x)=\frac{2}{\lambda} \phi(\phi(\lambda x))-x .
$$

(Gaidashev and Koch 2008) also suggests an analytic approach to the full period doubling problem for area-preserving maps based on its proximity to the one dimensional.

Infinitely renormalizable maps, that is maps on the renormalization stable manifold, have been studied in (Gaidashev and Johnson 2009) and (Gaidashev et al 2011), where it has been shown that such maps (in a codimension 1 stable submanifold) posses stable Cantor sets (sets on which the Lyapunov exponents 
are equal to zero) on which dynamics is rigid: the stable dynamics for two different infinitely renormalizable maps is conjugated by a $C^{1+\alpha}$ transformation (that is, a differentiable transformation whose derivative is Hölder with exponent $\alpha$ ). This is in a stark contrast to the situation in dissipative Hénon-like maps, where universality of Cantor sets (around the so called "tip") coexists with non-rigidity of the conjugacy of the dynamics on these sets (see (de Carvalho et al 2005, Lyubich and Martens 2008, Hazard et al 2010)).

The computer-assisted proof of (Eckmann et al 1984) can be described as an application of the Contraction Mapping Principle for a Newton map for the operator (1.1) in some neighborhood in an appropriately chosen Banach space. The proof is similar in spirit to the proofs (Lanford 1982, Lanford 1984) of the Feigenbaum-Coullet-Tresser universality by Oscar Lanford, and uses bounds for analytic functions (not necessarily polynomials) via the so called "standard sets": loosely, generalizations of intervals in $\mathbb{R}$ to infinite-dimensional functional spaces. Such computer assisted proofs came to be called "hard" within the computational community. The approach turned out to be quite powerful, albeit rather specialized and time and effort consuming.

In this paper we study analyticity and compactness of the renormalization operator (1.1) using, almost exclusively, the interval arithmetics in $\mathbb{R}$. We obtain a bound on the neighborhood of analyticity of the operator. Specifically, we identify a low order polynomial approximation for the "generating function" of the fixed point map of (1.1), and estimate the radius of the neighborhood of analyticity of the renormalization operator, centered on this polynomial approximation in an appropriate Banach space of functions analytic on a certain domain in $\mathbb{R}^{2}$.

Furthermore, we demonstrate that renormalizations of functions from this neighborhood of analyticity, are themselves functions, analytic on a larger domain in $\mathbb{R}^{2}$, which implies compactness of the renormalization operator.

We achieve these two goals using only what can be termed as "mild" computer assistance: computer estimates on arithmetic operations on reals and on the square root function on reals, as well as norm estimates of low order (quadratic) polynomials. It is also our hope that the reader can find of interest several little "tricks" used in the paper to get bounds on solutions of functional equations.

\section{A renormalization operator on generating functions}

An "area-preserving map" will mean an exact symplectic diffeomorphism of a subset of $\mathbb{R}^{2}$ onto its image.

Recall, that an area-preserving map $F:(x, u) \mapsto(y, v)$ can be uniquely specified by its generating function $\mathcal{S}$ :

$$
\left(\begin{array}{c}
x \\
-\mathcal{S}_{1}(x, y)
\end{array}\right) \stackrel{F}{\mapsto}\left(\begin{array}{c}
y \\
\mathcal{S}_{2}(x, y)
\end{array}\right), \quad \mathcal{S}_{i} \equiv \partial_{i} \mathcal{S},
$$

if the equation $u=-\mathcal{S}_{1}(x, y)$ has the unique solution $y(x, u)$. 
Furthermore, we will assume that $F$ is reversible, that is

$$
T \circ F \circ T=F^{-1}, \quad \text { where } T(x, u)=(x,-u) .
$$

For such maps it follows from (2.2) that

$$
\mathcal{S}_{1}(y, x)=\mathcal{S}_{2}(x, y) \equiv s(x, y),
$$

and

$$
\left(\begin{array}{c}
x \\
-s(y, x)
\end{array}\right) \stackrel{F}{\mapsto}\left(\begin{array}{c}
y \\
s(x, y)
\end{array}\right) .
$$

It is this "little" $s$ that will be referred to below as "the generating function". Applying a reversible $F$ twice we get

$$
\left(\begin{array}{c}
x^{\prime} \\
-s\left(z^{\prime}, x^{\prime}\right)
\end{array}\right) \stackrel{F}{\mapsto}\left(\begin{array}{c}
z^{\prime} \\
s\left(x^{\prime}, z^{\prime}\right)
\end{array}\right)=\left(\begin{array}{c}
z^{\prime} \\
-s\left(y^{\prime}, z^{\prime}\right)
\end{array}\right) \stackrel{F}{\mapsto}\left(\begin{array}{c}
y^{\prime} \\
s\left(z^{\prime}, y^{\prime}\right)
\end{array}\right) .
$$

It has been argued in (Collet et al 1981) that

$$
\Lambda_{F}(x, u)=(\lambda x, \mu u),
$$

where $\lambda$ and $\mu$ are some real parameters, dependent on $F$.

We therefore set $\left(x^{\prime}, y^{\prime}\right)=(\lambda x, \lambda y), z^{\prime}(\lambda x, \lambda y)=z(x, y)$ to obtain:

$$
\left(\begin{array}{c}
x \\
-\frac{1}{\mu} s(z, \lambda x)
\end{array}\right) \stackrel{\Lambda}{\mapsto}\left(\begin{array}{c}
\lambda x \\
-s(z, \lambda x)
\end{array}\right) \stackrel{F \circ F}{\mapsto}\left(\begin{array}{c}
\lambda y \\
s(z, \lambda y)
\end{array}\right) \stackrel{\Lambda^{-1}}{\mapsto}\left(\begin{array}{c}
y \\
\frac{1}{\mu} s(z, \lambda y)
\end{array}\right),
$$

where $z(x, y)$ solves

$$
s(\lambda x, z(x, y))+s(\lambda y, z(x, y))=0 .
$$

If the solution of (2.4) is unique, then $z(x, y)=z(y, x)$, and it follows from (2.3) that the generating function of the renormalized $F$ is given by

$$
\tilde{s}(x, y)=\mu^{-1} s(z(x, y), \lambda y) .
$$

Furthermore, it is possible to fix some normalization conditions for $\tilde{s}$ and $z$ which serve to determine scalings $\lambda$ and $\mu$ as functions of $s$. Notice, that the normalization

$$
s(1,0)=0
$$

is reproduced for $\tilde{s}$ as long as

$$
z(1,0)=z(0,1)=1 .
$$

In particular, this implies that

$$
s(\lambda, 1)+s(0,1)=0 .
$$


Furthermore, the condition

$$
\partial_{1} s(1,0)=1
$$

is reproduced as long as

$$
\mu=\partial_{1} z(1,0) .
$$

We will now summarize the above discussion in the following definition of the renormalization operator acting on generating functions originally due to the authors of (Eckmann et al 1984):

\section{Definition 2.1}

$$
\begin{aligned}
\mathcal{R}_{E K W}[s](x, y) & =\mu^{-1} s(z(x, y), \lambda y), \text { where } \\
0 & =s(\lambda x, z(x, y))+s(\lambda y, z(x, y)), \\
0 & =s(\lambda, 1)+s(0,1) \quad \text { and } \quad \mu=\partial_{1} z(1,0) .
\end{aligned}
$$

Definition 2.2 The Banach space of functions $s(x, y)=\sum_{i, j=0}^{\infty} c_{i j}(x-\tau)^{i}(y-$ $\tau)^{j}$, analytic on a bi-disk

$$
|x-\tau|<\rho,|y-\tau|<\rho,
$$

for which the norm

$$
\|s\|_{\rho}=\sum_{i, j=0}^{\infty}\left|c_{i j}\right| \rho^{i+j}
$$

is finite, will be referred to as $\mathcal{A}^{\tau}(\rho)$.

$\mathcal{A}_{s}^{\tau}(\rho)$ will denote its symmetric subspace $\left\{s \in \mathcal{A}^{\tau}(\rho): s_{1}(x, y)=s_{1}(y, x)\right\}$. We will also use the shorthand notation

$$
\mathcal{A}(\rho) \equiv \mathcal{A}^{0}(\rho), \quad \mathcal{A}_{s}(\rho) \equiv \mathcal{A}_{s}^{0}(\rho) .
$$

As we have already mentioned, the following theorem has been proved with the help of a computer in (Eckmann et al 1982) and (Eckmann et al 1984), and later in (Gaidashev et al 2011). We will quote here the version of the theorem from (Gaidashev et al 2011), since we will use the same functional spaces as the proofs in that paper.

Theorem 1 There exists a polynomial $s_{a}: \mathbb{C}^{2} \mapsto \mathbb{C}$, such that

i) The operator $\mathcal{R}_{E K W}$ is well-defined, analytic and compact in $\mathcal{B}_{r}\left(s_{0}\right) \subset$ $\mathcal{A}_{s}(\rho)$, with

$$
\rho=1.75, \quad r=1.1 \times 10^{-10} .
$$

ii) There exists a function $s^{*} \in \mathcal{B}_{r}\left(s_{0}\right) \subset \mathcal{A}_{s}(\rho)$ such that

$$
\mathcal{R}_{E K W}\left[s^{*}\right]=s^{*} \text {. }
$$


iii) The linear operator $D \mathcal{R}_{E K W}\left[s^{*}\right]$ has two eigenvalues outside of the unit circle:

$$
8.72021484375 \leq \delta_{1} \leq 8.72216796875, \quad \delta_{2}=\frac{1}{\lambda_{*}},
$$

where

$$
-0.248875313689 \leq \lambda_{*} \leq-0.248886108398438 .
$$

iv) The complement of these two eigenvalues in the spectrum is compactly contained in the unit disk. The largest eigenvalue in the unit disk is equal to $\lambda_{*}$, while

$$
\operatorname{spec}\left(D \mathcal{R}_{E K W}\left[s^{*}\right]\right) \backslash\left\{\delta_{1}, \delta_{2}, \lambda_{*}\right\} \subset\{z \in \mathbb{C}:|z| \leq 0.1258544921875\} .
$$

In this paper we will adopt a normalization condition different from (2.6) however. Specifically, $\mu$ will be defined from the normalization condition

$$
\mathcal{R}_{E K W}[s](0,0)=1,
$$

i.e

$$
\mu[s]=s(z(0,0), 0) .
$$

Definition 2.3 Define $\mathcal{I}^{\tau}(\rho)$ and $\mathcal{I}_{s}^{\tau}(\rho)$ to be the subsets of $\mathcal{A}^{\tau}(\rho)$ and $\mathcal{A}^{\tau}(\rho)$, respectively, of functions normalized in the following way

$$
\begin{aligned}
\mathcal{I}^{\tau}(\rho) & =\left\{s \in \mathcal{A}^{\tau}(\rho): s(0,0)=1\right\}, \\
\mathcal{I}_{s}^{\tau}(\rho) & =\left\{s \in \mathcal{A}_{s}^{\tau}(\rho): s(0,0)=1\right\}, \\
\mathcal{I}(\rho) & =\{s \in \mathcal{A}(\rho): s(0,0)=1\}, \\
\mathcal{I}_{s}(\rho) & =\left\{s \in \mathcal{A}_{s}(\rho): s(0,0)=1\right\} .
\end{aligned}
$$

Our main result concerning the operator 2.1 will be the following

Main Theorem 1 There exists a polynomial $s_{0} \in \mathcal{I}(\rho), \rho=1.75$, of degree 9 such that

i) the operator (2.1) is analytic in $B_{\delta}\left(s_{0}\right) \subset \mathcal{A}(\rho)$ with $\delta=0.00405550003051758$;

ii) for all $s \in B_{\delta}\left(s_{0}\right)$ with real Taylor coefficients, the scalings $\lambda=\lambda[s]$ and $\mu=\mu[s]$ satisfy

$$
\begin{aligned}
0.000406771898269653 & \leq \mu \leq 0.120654106140137 \\
-0.276069164276123 & \leq \lambda \leq-0.222213745117188
\end{aligned}
$$

iii) the operator (2.1) is compact, with $\mathcal{R}_{E K W}[s] \in \mathcal{I}\left(\rho^{\prime}\right), \rho^{\prime}=1.0699462890625 \rho$, for all $s \in B_{\delta}\left(s_{0}\right)$. 
Remark 2.4 We would like to note that the renormalization fixed point $s^{*}$ satisfies

$$
\left\|s^{*}-s_{0}\right\|_{\rho} \leq .00368565320968628,
$$

(which is a rigorous bound), i.e., as expected, according to Theorem 1, lies in the analyticity domain of renormalization.

Remark 2.5 All the numbers quoted in the Theorem and the Remark above are representable on a computer.

Although analyticity and compactness of the renormalization operator have been already proved in (Eckmann et al 1984) (together with a stronger result of existence of the fixed point), we would like to reiterate that the goal of the paper will be to obtain these results with a lighter machinery than that of (Eckmann et al 1984). We also obtain a better bound on the size of the neighborhood of analyticity of (2.1).

\section{Interval arithmetics in $\mathbb{R}$ and $\mathbb{R}^{2}$}

We will now give a very brief summary of interval operations in $\mathbb{R}$ and $\mathbb{R}^{2}$. For a more complete treatise of "standard sets" and operations on them, an interested reader is referred to an excellent review (Koch et al 1996).

A computer implementation of an arithmetic operation $r_{1} \# r_{2}$ (\# is,,$+- *$ or /) on two real numbers does not generally yield an exact result. The "computer" result is a number representable in a standard IEEE floating point format (cf (IEEE 1985)). Such numbers are commonly referred to as "representable". In the 80-bit extended precision IEEE arithmetics, a number is represented with 80 bits of memory: 1 bit for the sign of the number, 15 bits for an exponents, and 64 bits for a mantissa. A real representable number $r$ is of a the form

$$
r= \pm 1 . m 2^{e-\left(2^{14}-1\right)},
$$

where \pm is chosen according to whether the sign bit is 0 or $1, m$ is a base 2 mantissa given by the sequence of 0's and 1's associated with the 64 bits of the mantissa, and the exponent $0 \leq e \leq 32767$ is defined by the state of the 15 exponent bits (the state of 15 ones is reserved to represent "overflows"). The representable number 0 is given by the sequence of 80 zeros. The set of all representable numbers will be denoted $\mathcal{R}$.

Now, let $r_{1} \# r_{2}$ be a mathematically legal, nonzero, arithmetic operation. The result of the true arithmetic operation $r_{1} \# r_{2}$ might not be a representable number. However, we can instruct the computer to attempt to round this operation either to the nearest representable number, up or down (our choice was to always round up). This might not be possible: the result of rounding up might have the exponent $e \geq 32767$ (overflow), or $e<0$ (underflow). In both cases the computer is instructed to raise an exception, which is appropriately handled (either by terminating the program, or restarting with a different set of parameters). If, however, the result of rounding up is representable, the output of the 
computer implementation of the arithmetic operation is an upper bound on the true result of the operation. We will refer to such bound as "the upper bound".

We will define the standard sets in $\mathbb{R}$ to be the collection of all closed real intervals $I[x, y]=\{r \in \mathbb{R} \mid x \leq r \leq y\}$ :

$$
\operatorname{std}(\mathbb{R})=\{I[x, y] \in \mathbb{R}: x, y \in \mathcal{R}\} .
$$

If $I[x, y], I\left[x_{1}, y_{1}\right]$ and $I\left[x_{2}, y_{2}\right]$ are in $\operatorname{std}(\mathbb{R})$ then we can use the rounding up described above to obtain bounds on the arithmetic operation on these sets as follows:

1) unary minus: $-(I[x, y])=I[-y,-x]$;

2) absolute value: $|I[x, y]|=I[l, r]$, where $l=\max \{0, x,-y\}, r=-\min \{0, x,-y\}$;

3) addition: $I\left[x_{1}, y_{1}\right]+I\left[x_{2}, y_{2}\right]=I\left[x_{3}, y_{3}\right]$, where $y_{3}$ is the upper bound on $y_{1}+y_{2}$, while $-x_{3}$ is the upper bound on $-x_{1}+\left(-x_{2}\right)$;

4) subtraction: $I\left[x_{1}, y_{1}\right]-I\left[x_{2}, y_{2}\right] \equiv I\left[x_{1}, y_{1}\right]+\left(-I\left[x_{2}, y_{2}\right]\right)$;

5) multiplication: $I\left[x_{1}, y_{1}\right] \cdot I\left[x_{2}, y_{2}\right]=I\left[x_{3}, y_{3}\right]$, where $y_{3}$ is the maximum of the upper bounds on $x_{1} \cdot x_{2}, x_{1} \cdot y_{2}, y_{1} \cdot x_{2}$ and $y_{1} \cdot y_{2}$, while $-x_{3}$ is the maximum of the upper bounds on $\left(-x_{1}\right) \cdot x_{2},\left(-x_{1}\right) \cdot y_{2},\left(-y_{1}\right) \cdot x_{2}$ and $\left(-y_{1}\right) \cdot y_{2}$

6) inverse: if $x_{1} \cdot y_{1}>0$, then $I\left[x_{1}, y_{1}\right]$ inverse $=I\left[x_{2}, y_{2}\right]$, where $y_{2}$ is the upper bound on $1 / x_{1}$ and $-x_{2}$ is the upper bound on $1 /\left(-y_{1}\right)$;

7) division: if $x_{2} \cdot y_{2}>0$, then $I\left[x_{1}, y_{1}\right] / I\left[x_{2}, y_{2}\right] \equiv I\left[x_{1}, y_{1}\right] \cdot\left(I\left[x_{2}, y_{2}\right]\right.$ inverse).

A standard set in $\mathbb{R}^{2}$ is, naturally, a direct product of two standard sets in $\mathbb{R}$ :

$$
\operatorname{std}\left(\mathbb{R}^{2}\right)=\left\{I\left[x_{1}, y_{1}\right] \times I\left[x_{2}, y_{2}\right], x_{1}, x_{2}, y_{1}, y_{2} \in \mathcal{R}\right\} .
$$

The arithmetic operation on these standard sets in $\mathbb{R}^{2}$ are reducible to those on reals in an obvious way.

To obtain a bound on a algebraic and transcendental function, one can use their Taylor series together with a bound on the remainder. In our proofs we will require only three such functions: exp, $\ln$ and $\sqrt{ }$. All of them are implemented via a finite truncation of their Taylor series with a bound on the remainder.

\section{Contraction Mapping Principle}

We will now outline a rather general method for finding a fixed point of a hyperbolic operator in a Banach space via its approximate Newton map.

Let $C$ be an operator analytic and hyperbolic on some neighborhood $\mathcal{N}$ in a Banach space $\mathcal{Z}$. Suppose, that one knows its approximate hyperbolic fixed point $Z_{0} \in \mathcal{N}$. Set

$$
M \equiv\left[\mathbb{I}-D C\left[Z_{0}\right]\right]^{-1},
$$


and for all $z$, such that $Z_{0}+M z \in \mathcal{N}$,

$$
N[z]=z+C\left[Z_{0}+M z\right]-\left(Z_{0}+M z\right) .
$$

Notice, that if $z^{*}$ is a fixed point of $N$, then $Z_{0}+M z^{*}$ is a fixed point of $C$.

The linear operator $\mathbb{I}-D C\left[Z_{0}\right]$ is indeed invertible since $D C$ is hyperbolic at $Z_{0}$. If $Z_{0}$ is a reasonably good approximation of the true fixed point of $C$, then the operator $N$ is expected to be a strong contraction in a neighborhood of 0 :

$$
\begin{aligned}
D N[z] & =\mathbb{I}+D C\left[Z_{0}+M z\right] \cdot M-M \\
& =\left[M^{-1}+D C\left[Z_{0}+M z\right]-\mathbb{I}\right] \cdot M \\
& =\left[\mathbb{I}-D C\left[Z_{0}\right]+D C\left[Z_{0}+M z\right]-\mathbb{I}\right] \cdot M \\
& =\left[D C\left[Z_{0}+M z\right]-D C\left[Z_{0}\right]\right] \cdot M .
\end{aligned}
$$

The last expression is typically small in a small neighborhood of 0 , if the norm of $M$ is not too large (if $M$ is large, one might have to find a better approximation $Z_{0}$ and take a smaller neighborhood of 0 ). The following well-known Theorem specifies a sufficient condition for existence of the fixed point:

Theorem 4.1 (Contraction Mapping Principle)

Suppose that the operator $N$ is well-defined and analytic as a map from $\mathcal{N} \subset \mathcal{Z}$ to $\mathcal{Z}$, where $\mathcal{Z}$ is some Banach space. Let $Z_{0} \in \mathcal{N}$ and $B_{\delta}\left(Z_{0}\right) \subset \mathcal{N}$ (an open ball of radius $\delta$ around $Z_{0}$ ) be such that

$$
\|D N[Z]\| \leq \mathcal{D}<1
$$

for any $Z \in B_{\delta}\left(Z_{0}\right)$, and

$$
\left\|N\left[Z_{0}\right]-Z_{0}\right\| \leq \epsilon
$$

If $\epsilon<(1-\mathcal{D}) \delta$ then the operator $N$ has a fixed point $Z^{*}$ in $B_{\delta}\left(Z_{0}\right)$, such that

$$
\left\|Z^{*}-Z_{0}\right\| \leq \frac{\epsilon}{1-\mathcal{D}} .
$$

We will use the above Contraction Mapping Principle in several instances in our proofs below. In all those cases we will be verifying the hypothesis of the Contraction Mapping Principle using mild computer assistance.

\section{Analyticity of renormalization}

We consider the space $\mathcal{A}(\rho), \rho=1.75$. Define

$$
s_{0}(x, y) \equiv D_{0}(x) y^{3}+A_{0}(x) y^{2}+B_{0}(x) y+C_{0}(x),
$$

a polynomial of degree 3 , with

$$
D_{0}(x)=\sum_{i=0}^{6} d_{i} x^{i}, \quad A_{0}(x)=\sum_{i=0}^{6} a_{i} x^{i}, \quad B_{0}(x)=\sum_{i=0}^{6} b_{i} x^{i}, \quad C_{0}(x)=\sum_{i=0}^{6} c_{i} x^{i},
$$

where the coefficients of these polynomials are as follows (the numbers given in the table are highly accurate approximations of the representable numbers actually used in the programs): 


\begin{tabular}{|c|c|r|}
\hline$i$ & $c_{i}$ & $b_{i}$ \\
\hline 0 & 1.00000000000000000 & $-2.42962369607899157 \times 10^{-1}$ \\
\hline 1 & -1.02761956458970711 & $5.87327440047455615 \times 10^{-2}$ \\
\hline 2 & $2.93663720023727808 \times 10^{-2}$ & $-5.93710236103475834 \times 10^{-3}$ \\
\hline 3 & $-1.87658664952086400 \times 10^{-3}$ & $6.09332694202817819 \times 10^{-4}$ \\
\hline 4 & $1.40668294317213841 \times 10^{-4}$ & $-6.46957663100331420 \times 10^{-5}$ \\
\hline 5 & $-1.18664608613747513 \times 10^{-5}$ & $7.02844653606969302 \times 10^{-6}$ \\
\hline 6 & $1.06935654680404746 \times 10^{-6}$ & $-7.75814237637266867 \times 10^{-7}$ \\
\hline \hline$i$ & $a_{i}$ & $d_{i}$ \\
\hline 0 & $-8.77647505670140721 \times 10^{-1}$ & $-1.46791670728014469 \times 10^{-3}$ \\
\hline 1 & $-5.62975994856259201 \times 10^{-3}$ & $5.62673177268855366 \times 10^{-4}$ \\
\hline 2 & $9.13999041304226728 \times 10^{-4}$ & $-1.29391532620066284 \times 10^{-4}$ \\
\hline 3 & $-1.33251463600020727 \times 10^{-4}$ & $2.45371180652649971 \times 10^{-5}$ \\
\hline 4 & $1.84028385489487478 \times 10^{-5}$ & $-4.18641657324405651 \times 10^{-6}$ \\
\hline 5 & $-2.46417657757601024 \times 10^{-6}$ & $6.68319468409332288 \times 10^{-7}$ \\
\hline 6 & $3.23459112039180563 \times 10^{-7}$ & $-1.01939399249366523 \times 10^{-7}$. \\
\hline
\end{tabular}

A numerical study demonstrates that $s_{0}$ is a relatively good approximation of the renormalization fixed point in $\mathcal{A}_{s}(\rho)$ for a rather wide range of radii $\rho$ ( $\rho \approx 0.8 \ldots 2.5$, below $\rho$ will be fixed to be 1.75$)$.

In this Section we will prove Theorem 1, This Theorem is proved with a mild aid of the computer. The computer assistance is restricted only to interval arithmetics on real numbers, (which, given sufficient time, in principle, can be done "by hand").

We will start by noticing that a solution of the equation

$$
s\left(x, Z^{\prime}\right)+s\left(y, Z^{\prime}\right)=0
$$

with $s \equiv s_{0}$ is given by

$$
Z_{0}^{\prime}(x, y)=\frac{B_{0}(x)+B_{0}(y)}{2\left(A_{0}(x)+A_{0}(y)\right)}\left[\sqrt{1-4 \frac{\left(A_{0}(x)+A_{0}(y)\left(\tilde{C}_{0}(x)+\tilde{C}_{0}(y)\right.\right.}{\left(B_{0}(x)+B_{0}(y)\right)^{2}}}-1\right]
$$

where $\tilde{C}_{0}(x)$ is a bound on $C_{0}(x)+y^{3} D(x)$, specifically:

$$
\tilde{C}_{0}(x)=C_{0}(x)+C D_{0}(x), \quad C \in \mathbb{D}_{\rho^{3}}(0) .
$$

In the formula above we choose a branch of $\sqrt{ }$ such that the range of $Z_{0}^{\prime}$ is expected to be contained in $D_{\rho}$.

We would like to remark that we have intentionally chosen a polynomial $s_{0}$ of a sufficiently high degree so that it would be close to the fixed point $s^{*}$ found in (Gaidashev et al 2011). This will later imply that the fixed point $s^{*}$ is indeed in the domain of analyticity and compactness of $\mathcal{R}_{E K W}$. At the same time, taking a polynomial of degree higher than 3 in $y$ is superfluous for the purposes of the 
computation of $Z^{\prime}$. Therefore, these extra degrees (polynomial $D_{0}$ ) have been included as an "error term" in (5.10). It is clear, that

$$
C_{0}(x)+y^{3} D_{0}(x) \in \tilde{C}_{0}(x)
$$

for all $(x, y)$ such that $|x|<\rho,|y|<\rho$.

We shall now check that for the given values of coefficients the function $Z_{0}^{\prime}$ is analytic on a bi-disk of radius $r=0.483119964599609$ centered at point $(0,0)$. Below $\mathcal{Z}(r)$ will denote the Banach space of functions analytic on $\{(x, y):|x|<r,|y|<r\}$ equipped with the sup-norm, denoted by $|\cdot|_{r}$. We have the following

Lemma $5.1 Z_{0}^{\prime}$ is in $\mathcal{Z}(r)$ for $r=0.483119964599609$, and satisfies

$$
\left|Z_{0}^{\prime}\right|_{r}<1.562789916992188 .
$$

Proof. First, define constants $v_{0}, \beta_{0}$ and $\gamma_{0}$, and quartic polynomials $v, \beta$ and $\gamma$, such that $v(0,0)=\beta(0,0)=\gamma(0,0)=0$ by setting

$$
\begin{aligned}
v_{0}+v(x, y) & =A_{0}(x)+A_{0}(y) \\
\beta_{0}+\beta(x, y) & =B_{0}(x)+B_{0}(y), \\
\gamma_{0}+\gamma(x, y) & =\tilde{C}_{0}(x)+\tilde{C}_{0}(y) .
\end{aligned}
$$

Then

$$
Z_{0}^{\prime}(x, y)=\frac{\beta_{0}+\beta(x, y)}{2\left(v_{0}+v(x, y)\right)}\left[\sqrt{1-4 \frac{v_{0} \gamma_{0}}{\beta_{0}^{2}}+F_{1}(x, y)-F_{2}(x, y)}-1\right],
$$

where

$$
\begin{aligned}
F_{1}(x, y) & =\frac{4 v_{0} \gamma_{0}}{\beta_{0}^{2}} \frac{2 \beta_{0} \beta(x, y)+\beta^{2}(x, y)}{\beta_{0}^{2}+2 \beta_{0} \beta(x, y)+\beta^{2}(x, y)} \\
F_{2}(X, Y) & =4 \frac{v_{0} \gamma(x, y)+v(x, y) \gamma_{0}+v(x, y) \gamma(x, y)}{\beta_{0}^{2}+2 \beta_{0} \beta(x, y)+\beta^{2}(x, y)} .
\end{aligned}
$$

Norms of both $F_{1}$ and $F_{2}$ are elementary:

$$
\left|F_{1}\right|_{r} \leq \frac{4\left|v_{0} \gamma_{0}\right|}{\beta_{0}^{2}} \frac{\left|2 \beta_{0} \beta+\beta^{2}\right|_{r}}{\beta_{0}^{2}-\left|2 \beta_{0} \beta+\beta^{2}\right|_{r}}, \quad\left|F_{2}\right|_{r} \leq 4 \frac{\left|v_{0} \gamma+v \gamma_{0}+v \gamma\right|_{r}}{\beta_{0}^{2}-\left|2 \beta_{0} \beta+\beta\right|_{r}^{2}} .
$$

Notice that all functions here whose norm has to be evaluated are explicit polynomials. We have estimated the above norms using the interval arithmetics on a computer. Next, we use these bounds in the following expression

$$
\left|Z_{0}^{\prime}\right|_{r} \leq \frac{\left|\beta_{0}\right|+|\beta|_{r}}{2\left(\left|v_{0}\right|-|v|_{r}\right)} \max _{j= \pm 1}\left\{\sqrt{1-4 \frac{v_{0} \gamma_{0}}{\beta_{0}^{2}}+j\left|F_{1}\right|_{r}+j\left|F_{2}\right|_{r}}-1\right\},
$$

and evaluate it on a computer. 
Below, we will use the following shorthand notation for the supremum of $Z_{0}^{\prime}$ :

$$
t \equiv\left|Z_{0}^{\prime}\right|_{r}
$$

Notice that if one writes

$$
s(x, y) \equiv \sigma(x, y)-\frac{\tau(y)}{2},
$$

where $\tau$ is chosen so that it has an inverse branch $\nu$ with a range in $D_{\rho}$ (it is sufficient to choose $\tau$ a quadratic function), then a solution of equation (51) satisfies

$$
Z^{\prime}(x, y)=\nu\left[\sigma\left(x, Z^{\prime}(x, y)\right)+\sigma\left(y, Z^{\prime}(x, y)\right)\right] .
$$

(this "trick" has been shown to me by Hans Koch).

We will now specify the choices of $\tau$ and $\sigma$ for $s_{0}$. Set

$$
\begin{aligned}
s_{0}(x, y) & \equiv \sigma_{0}(x, y)-\frac{\tau(y)}{2}, \\
\sigma_{0}(x, y) & \equiv a(x) y^{2}+b(x) y+c(x), \quad \text { and } \quad \Sigma_{0}(x, y)=\sigma_{0}(x+p, y), \\
\tau(y) & \equiv-2 a_{0} y^{2}-2 b_{0} y, \\
\nu(x) & \equiv \frac{-b_{0}+\sqrt{b_{0}^{2}-2 a_{0} x}}{2 a_{0}} .
\end{aligned}
$$

It can be readily verified that $Z_{0}^{\prime}(x, y)=\nu\left[\Sigma_{0}\left(x, Z_{0}^{\prime}(x, y)\right)+\Sigma_{0}\left(y, Z_{0}^{\prime}(x, y)\right)\right]$. We will next demonstrate that the operator $C_{h}$, defined on $\mathcal{Z}(r)$ by setting

$$
C_{h}\left[Z^{\prime}\right] \equiv \nu\left[\Sigma_{0} \circ\left(\Pi_{1}, Z^{\prime}\right)+h \circ\left(P_{1}, Z^{\prime}\right)+\Sigma_{0} \circ\left(\Pi_{2}, Z^{\prime}\right)+h \circ\left(P_{2}, Z^{\prime}\right)\right],
$$

where

$$
P_{1}(x, y) \equiv x+p, \quad \text { and } \quad P_{2}(x, y) \equiv y+p,
$$

has a fixed point for sufficiently small $h$ 's.

Proposition 5.2 Let $\delta, \rho$ and $r$ be as in Theorem 11 and Lemma 5.1. Then, for all $s \in B_{\delta}\left(s_{0}\right) \subset \mathcal{A}(\rho)$ the operator $C_{s-s_{0}}$ has a unique fixed point $Z_{s}^{\prime}$ in $B_{\epsilon}\left(Z_{0}^{\prime}\right) \subset \mathcal{Z}(r)$ with $\epsilon=0.01465$, and the map $s \mapsto Z_{s}^{\prime}$ is analytic from $B_{\delta}\left(s_{0}\right)$ to $B_{\epsilon}\left(Z_{0}^{\prime}\right)$.

Proof. Define an operator

$$
N_{h}[z]=z+C_{h}\left[Z_{0}^{\prime}+M z\right]-\left(Z_{0}^{\prime}+M z\right), \quad M \equiv\left[\mathbb{I}-D C_{0}\left[Z_{0}^{\prime}\right]\right]^{-1} .
$$

We will demonstrate that this "Newton map" has a fixed point in a neighborhood of 0 . 
We will first estimate the norm of $N_{h}[0]=C_{h}\left[Z_{0}^{\prime}\right]-Z_{0}^{\prime}$ :

$$
\begin{aligned}
&\left|C_{h}\left[Z_{0}^{\prime}\right]-Z_{0}^{\prime}\right|_{r}= \mid \nu\left[\Sigma_{0} \circ\left(\Pi_{1}, Z_{0}^{\prime}\right)+h \circ\left(\Pi_{1}, Z_{0}^{\prime}\right)+\Sigma_{0} \circ\left(\Pi_{2}, Z_{0}^{\prime}\right)+h \circ\left(\Pi_{2}, Z_{0}^{\prime}\right)\right] \\
& \quad-\left.\nu\left[\Sigma_{0}\left(\Pi_{1}, Z_{0}^{\prime}\right)+\Sigma_{0}\left(\Pi_{2}, Z_{0}^{\prime}\right)\right]\right|_{r} \\
&= \frac{1}{2 a_{0}} \mid \sqrt{b_{0}^{2}-2 a_{0}\left[\Sigma_{0}\left(\Pi_{1}, Z_{0}^{\prime}\right)+h\left(P_{1}, Z_{0}^{\prime}\right)+\Sigma_{0}\left(\Pi_{2}, Z_{0}^{\prime}\right)+h\left(P_{2}, Z_{0}^{\prime}\right)\right]} \\
& \quad \quad-\left.\sqrt{b_{0}^{2}-2 a_{0}\left[\Sigma_{0}\left(\Pi_{1}, Z_{0}^{\prime}\right)+\Sigma_{0}\left(\Pi_{2}, Z_{0}^{\prime}\right)\right]}\right|_{r} \\
&=\frac{1}{2 a_{0}}|F(1)-F(0)|_{r},
\end{aligned}
$$

where

$$
F(\zeta)=\sqrt{b_{0}^{2}-2 a_{0}\left[\Sigma_{0}\left(\Pi_{1}, Z_{0}^{\prime}\right)+\zeta h\left(P_{1}, Z_{0}^{\prime}\right)+\Sigma_{0}\left(\Pi_{2}, Z_{0}^{\prime}\right)+\zeta h\left(P_{2}, Z_{0}^{\prime}\right)\right]}
$$

is an analytic function from $\mathbb{D}_{R}$ to $\mathcal{Z}(r)$ (here and below, $\mathbb{D}_{R}$ stands for an open disk of radius $R$ in $\mathbb{C}$ ), with

$$
R \leq \frac{b_{0}^{2}-4 a_{0} \theta_{0}-2\left|a_{0}\right|\left|\Theta_{0}\left(\Pi_{1}, Z_{0}^{\prime}\right)\right|_{r}-2\left|a_{0}\right|\left|\Theta_{0}\left(\Pi_{2}, Z_{0}^{\prime}\right)\right|_{r}}{2\left|a_{0}\right|\left|h\left(P_{1}, Z_{0}^{\prime}\right)\right|_{r}+2\left|a_{0}\right|\left|h\left(P_{2}, Z_{0}^{\prime}\right)\right|_{r}},
$$

where

$$
\theta_{0} \equiv \Sigma_{0}(0,0), \quad \Theta_{0}(x, y) \equiv \Sigma_{0}(x, y)-\theta_{0} .
$$

Below, we will denote the (positive) real number $b_{0}^{2}-4 a_{0} \theta_{0}$ by $c$. A straightforward Cauchy estimate yields:

$$
|F(1)-F(0)| \leq \frac{1}{R-1} \sup _{|\zeta| \leq R}|F(\zeta)| \leq \frac{4\left|a_{0}\right| \delta \sqrt{2 c}}{c-4\left|a_{0}\right|\left|\Theta_{0}\right|_{(r, t)}-4\left|a_{0}\right| \delta} .
$$

We will denote

$$
\varepsilon \equiv \frac{2 \delta \sqrt{2 c}}{c-4\left|a_{0}\right|\left|\Theta_{0}\right|_{(r, t)}-4\left|a_{0}\right| \delta} .
$$

This expression has been shown to satisfy

$$
\varepsilon<0.0137615203857422 \text {. }
$$

As a next step we will provide a bound on the derivative of the operator $N_{h}$ :

$$
\begin{aligned}
\left|D N_{h}[z]\right| & =\left|\left[D C_{h}\left[Z_{0}^{\prime}+M z\right]-D C_{0}\left[Z_{0}^{\prime}\right]\right] M\right| \\
& \leq\left|\left[D C_{h}\left[Z_{0}^{\prime}+M z\right]-D C_{0}\left[Z_{0}^{\prime}\right]\right]\right||M| .
\end{aligned}
$$

To estimate the norm of $M$ we first verify that $\left|D C_{0}\left[Z_{0}^{\prime}\right]\right|<1$ :

$$
\begin{aligned}
\left|D C_{0}\left[Z_{0}^{\prime}\right]\right| & =\left|\frac{\partial_{2} \Theta_{0}\left(\Pi_{1}, Z_{0}^{\prime}\right)+\partial_{2} \Theta_{0}\left(\Pi_{2}, Z_{0}^{\prime}\right)}{2 \sqrt{c-2 a\left[\Theta_{0}\left(\Pi_{1}, Z_{0}^{\prime}\right)+\Theta_{0}\left(\Pi_{2}, Z_{0}^{\prime}\right)\right]}}\right| \\
& \leq \frac{\left|\partial_{2} \Theta_{0}\right|_{(r, t)}}{\sqrt{c-4\left|a_{0}\right|\left|\Theta_{0}\right|_{(r, t)}}} .
\end{aligned}
$$


A computer-aided evaluation of the last expression indeed shows that it is less than 1. This implies that $M$ can be found as the limit of a convergent series whose norm is easily bounded:

$$
|M| \leq \frac{1}{1-\left|D C_{0}\left[Z_{0}^{\prime}\right]\right|}<1.0430755615234375 \equiv \mathcal{M} .
$$

Next,

$$
\begin{aligned}
D C_{h}\left[Z^{\prime}\right]-D C_{0}\left[Z_{0}^{\prime}\right] & =\frac{\partial_{2} \Theta_{0}\left(\Pi_{1}, Z^{\prime}\right)+\partial_{2} \Theta_{0}\left(\Pi_{2}, Z^{\prime}\right)+\partial_{2} h\left(P_{1}, Z^{\prime}\right)+\partial_{2} h\left(P_{2}, Z^{\prime}\right)}{2 \sqrt{c-2 a_{0}\left[\Theta_{0}\left(\Pi_{1}, Z^{\prime}\right)+\Theta_{0}\left(\Pi_{2}, Z^{\prime}\right)+h\left(P_{1}, Z^{\prime}\right)+h\left(P_{2}, Z^{\prime}\right)\right]}} \\
& -\frac{\partial_{2} \Theta_{0}\left(\Pi_{1}, Z_{0}^{\prime}\right)+\partial_{2} \Theta_{0}\left(\Pi_{2}, Z_{0}^{\prime}\right)}{2 \sqrt{c-2 a_{0}\left[\Theta_{0}\left(\Pi_{1}, Z_{0}^{\prime}\right)+\Theta_{0}\left(\Pi_{2}, Z_{0}^{\prime}\right)\right]}} \\
& =\left[\frac{\partial_{2} \Theta_{0}\left(\Pi_{1}, Z^{\prime}\right)+\partial_{2} \Theta_{0}\left(\Pi_{2}, Z^{\prime}\right)+\partial_{2} h\left(P_{1}, Z^{\prime}\right)+\partial_{2} h\left(P_{2}, Z^{\prime}\right)}{2 \sqrt{c-2 a_{0}\left[\Theta_{0}\left(\Pi_{1}, Z^{\prime}\right)+\Theta_{0}\left(\Pi_{2}, Z^{\prime}\right)+h\left(P_{1}, Z^{\prime}\right)+h\left(P_{2}, Z^{\prime}\right)\right]}}\right. \\
& \left.-\frac{\partial_{2} \Theta_{0}\left(\Pi_{1}, Z^{\prime}\right)+\partial_{2} \Theta_{0}\left(\Pi_{2}, Z^{\prime}\right)}{2 \sqrt{c-2 a_{0}\left[\Theta_{0}\left(\Pi_{1}, Z^{\prime}\right)+\Theta_{0}\left(\Pi_{2}, Z^{\prime}\right)+h\left(P_{1}, Z^{\prime}\right)+h\left(P_{2}, Z^{\prime}\right)\right]}}\right] \\
& +\left[\frac{\partial_{2} \Theta_{0}\left(\Pi_{1}, Z^{\prime}\right)+\partial_{2} \Theta_{0}\left(\Pi_{2}, Z^{\prime}\right)}{2 \sqrt{c-2 a_{0}\left[\Theta_{0}\left(\Pi_{1}, Z^{\prime}\right)+\Theta_{0}\left(\Pi_{2}, Z^{\prime}\right)+h\left(P_{1}, Z^{\prime}\right)+h\left(P_{2}, Z^{\prime}\right)\right]}}\right. \\
& \left.-\frac{\partial_{2} \Theta_{0}\left(\Pi_{1}, Z^{\prime}\right)+\partial_{2} \Theta_{0}\left(\Pi_{2}, Z^{\prime}\right)}{2 \sqrt{c-2 a_{0}\left[\Theta_{0}\left(\Pi_{1}, Z^{\prime}\right)+\Theta_{0}\left(\Pi_{2}, Z^{\prime}\right)\right]}}\right] \\
& +\left[\frac{\partial_{2} \Theta_{0}\left(\Pi_{1}, Z^{\prime}\right)+\partial_{2} \Theta_{0}\left(\Pi_{2}, Z^{\prime}\right)}{2 \sqrt{c-2 a_{0}\left[\Theta_{0}\left(\Pi_{1}, Z^{\prime}\right)+\Theta_{0}\left(\Pi_{2}, Z^{\prime}\right)\right]}}\right. \\
& \left.-\frac{\partial_{2} \Theta_{0}\left(\Pi_{1}, Z_{0}^{\prime}\right)+\partial_{2} \Theta_{0}\left(\Pi_{2}, Z_{0}^{\prime}\right)}{2 \sqrt{c-2 a_{0}\left[\Theta_{0}\left(\Pi_{1}, Z_{0}^{\prime}\right)+\Theta_{0}\left(\Pi_{2}, Z_{0}^{\prime}\right)\right]}}\right] \\
& \equiv I_{1}+I_{2}+I_{3} .
\end{aligned}
$$

We will estimate norms of the three expressions in brackets separately.

$$
\left|I_{1}\right|_{r} \leq \frac{\left|\partial_{2} h\right|_{(r, s)}}{\sqrt{c-4\left|a_{0}\right|\left|\Theta_{0}\right|_{(r, s)}-4\left|a_{0}\right||h|_{(r, s)}}} \leq \frac{m \delta}{\sqrt{c-4\left|a_{0}\right|\left|\Theta_{0}\right|_{(r, s)}-4\left|a_{0}\right| \delta}}
$$

where

$$
s=t+|M| \epsilon, \quad m=n \frac{s^{n-1}}{\rho^{n}} \quad \text { and } \quad n=\frac{1}{\ln \left[\frac{\rho}{s}\right]} .
$$

We use a Cauchy estimate for the function

$$
F_{2}(\zeta)=\frac{1}{\sqrt{c-2 a_{0}\left[\Theta_{0}\left(\Pi_{1}, Z^{\prime}\right)+\Theta_{0}\left(\Pi_{2}, Z^{\prime}\right)+\zeta h\left(P_{1}, Z^{\prime}\right)+\zeta h\left(P_{2}, Z^{\prime}\right)\right]}}
$$

to bound $\left|I_{2}\right|_{r}$. Notice, that, $F_{2}$ is also analytic on $\mathbb{D}_{R}$, but gets unbounded as $\zeta$ approaches the boundary of this disk. Therefore, to bound $\left|I_{2}\right|_{r}$, we use a Cauchy bound for $F_{2}$ on a smaller disk of radius 


$$
\begin{gathered}
\tilde{R}=\frac{c-4\left|a_{0}\right|\left|\Theta_{0}\right|_{(r, s)}}{4\left|a_{0}\right| \delta}-\frac{1}{3} \frac{c-4\left|a_{0}\right|\left|\Theta_{0}\right|_{(r, s)}-4\left|a_{0}\right| \delta}{4\left|a_{0}\right| \delta}: \\
\left|I_{2}\right|_{r} \leq\left|\partial_{2} \Theta_{0}\right|_{(r, s)} \frac{1}{\tilde{R}-1} \sup _{|\zeta| \leq \tilde{R}}\left|F_{2}(\zeta)\right| \leq \frac{4\left|a_{0}\right| \delta\left|\partial_{2} \Theta_{0}\right|_{(r, s)}}{2\left(\frac{1}{3}\left(c-4\left|a_{0}\right|\left|\Theta_{0}\right|_{(r, s)}-4\left|a_{0}\right| \delta\right)\right)^{3 / 2}} .
\end{gathered}
$$

Finally, to estimate $\left|I_{3}\right|_{r}$, we use a Cauchy bound for the function

$$
F_{3}(\zeta)=\frac{\partial_{2} \Theta_{0}\left(\Pi_{1}, Z_{0}^{\prime}+\zeta M z\right)+\partial_{2} \Theta_{0}\left(\Pi_{2}, Z_{0}^{\prime}+\zeta M z\right)}{2 \sqrt{c-2 a_{0}\left[\Theta_{0}\left(\Pi_{1}, Z_{0}^{\prime}+\zeta M z\right)+\Theta_{0}\left(\Pi_{2}, Z_{0}^{\prime}+\zeta M z\right)\right]}},
$$

analytic on $\mathbb{D}_{\hat{R}}$ with

$$
\begin{gathered}
\hat{R}=\frac{\rho-t}{|M| \epsilon}: \\
\left|I_{3}\right|_{r}=\left|F_{3}(1)-F_{3}(0)\right| \leq \frac{1}{\hat{R}-1} \sup _{|\zeta| \leq \hat{R}}\left|F_{3}(\zeta)\right| \leq \frac{|M| \epsilon}{\rho-s} \frac{\left|\partial_{2} \Theta_{0}\right|_{(r, \rho)}}{\sqrt{c-4\left|a_{0}\right|\left|\Theta_{0}\right|_{(r, \rho)}}} .
\end{gathered}
$$

Individual norms $|M|,\left|I_{1}\right|_{r},\left|I_{2}\right|_{r}$ and $\left|I_{3}\right|_{r}$ and their sum have been estimated on a computer to produce:

$$
\left|D N_{h}(z)\right| \leq 0.0125999450683594 \equiv \mathcal{D}
$$

for all $\|h\|_{\rho}<\delta$ and $|z|_{r}<\epsilon$.

Estimates (5.14) and (5.19) demonstrate that

$$
\varepsilon<(1-\mathcal{D}) \frac{\epsilon}{\mathcal{M}},
$$

and the claim follows from the Contraction Mapping Principle.

At the next step we obtain bounds on the scaling parameters $\lambda$ and $\mu$.

Recall that the scaling $\lambda$ solves equation (2.6).

The prove Theorem 1 we will require the following

Lemma 5.3 Equation (2.6) has a solution $\lambda=\lambda[s]$ for all $s \in B_{\delta}\left(s_{0}\right)$, and satisfies

$$
-0.276069164276123 \leq \lambda \leq-0.222213745117188
$$

for all $s \in B_{\delta}\left(s_{0}\right)$ with real Taylor coefficients.

Furthermore, the map $s \mapsto \lambda[s]$ is analytic on $B_{\delta}\left(s_{0}\right)$.

Proof. Write,

$$
s_{1}(x, y) \equiv A_{1}(y) x^{2}+B_{1}(y) x+C_{1}(y)
$$


where

$$
\begin{aligned}
& A_{1}(y)=c_{2}+b_{2} y+a_{2} y^{2}+d_{2} y^{3}, \\
& B_{1}(y)=c_{1}+b_{1} y+a_{1} y^{2}+d_{1} y^{3}, \\
& C_{1}(y)=c_{0}+b_{0} y+a_{0} y^{2}+d_{0} y^{3} .
\end{aligned}
$$

Denote

Notice, that the equation (2.6) with $s=s_{1}$ can be solved exactly (a quadratic equation for $\lambda)$. Furthermore, suppose $s=s_{1}+h,\|h\|_{\rho} \leq\left\|s_{0}-s_{1}\right\|_{\rho}+\delta \equiv \tilde{\delta}$, then $\lambda[s]$ solves

$$
s_{1}(\lambda[s], 1)+s_{1}(0,1)=-h(\lambda[s], 1)-h(0,1) .
$$

Write

$$
\lambda[s]=\lambda\left[s_{1}\right]+\Delta \lambda,
$$

then $\Delta \lambda$ is a solution of

$$
A_{1}(1) \cdot(\Delta \lambda)^{2}+\left[2 A_{1}(1) \cdot \lambda\left[s_{1}\right]+B_{1}(1)\right] \cdot \Delta \lambda+C=0
$$

with $C \in \mathbb{D}_{2 \tilde{\delta}}$ (an open disk of radius $2 \tilde{\delta}$ in $\mathbb{C}$ ). For all $s$ with real coefficients, (5.21) is a quadratic equation for $\Delta \lambda$ with real coefficients, whose solution gives the required bound.

The last ingredient in the proof of analyticity of renormalization is a bound on the scaling $\mu$.

Lemma 5.4 Let $\mu[s]$ be as in equation (2.7). Then the map $s \mapsto \mu[s]$ is analytic on $B_{\delta}\left(s_{0}\right)$, and $\mu[s]$ satisfies

$$
0.000406771898269653 \leq \mu \leq 0.120654106140137,
$$

for $s \in B_{\delta}\left(s_{0}\right)$ with real Taylor coefficients.

Proof. The bound is straightforward:

$$
\begin{aligned}
\mu[s] & =s\left(Z_{s}^{\prime}(0,0), 0\right) \\
& \in s_{0}\left(Z_{s}^{\prime}(0,0), 0\right)+h\left(Z_{s}^{\prime}(0,0), 0\right) .
\end{aligned}
$$

Now, it is clear, that whenever $s$ has real coefficients,

$$
\mu[s] \in s_{0}\left(Z_{0}^{\prime}(0,0)+I[-|M| \epsilon,|M| \epsilon], 0\right)+I[-\delta, \delta],
$$

where, as before, $I[x, y]$ stands for a closed real interval with endpoints $x$ and $y$, while the rest of the quantities are as in Lemma 5.3 and Prop. 5.2 ,

Now, part $i$ ) of Theorem 10 follows immediately from Proposition 5.2 and Lemmas 5.3 and 5.4 


\section{Compactness of renormalization}

We will now outline the proof of compactness of the renormalization operator.

Proposition 6.1 For every $s \in B_{\delta}\left(s_{0}\right) \subset \mathcal{A}(\rho)$ its renormalization $\mathcal{R}_{E K W}[s]$ is in $\mathcal{I}(\kappa \rho)$ with $\kappa=1.0699996948242188$.

Proof. Let $r^{\prime}=\kappa r$ and $\rho^{\prime}=\kappa \rho$. Verification that $|\lambda[s]| \rho^{\prime} \mid \leq \rho$ is straightforward. The "difficult" part is to demonstrate that $\left|Z_{s}^{\prime}\right|_{r^{\prime}} \leq \rho$. To show this, we will use the fact that $Z_{s}^{\prime}=\lim _{n \rightarrow \infty} N_{s-s_{0}}[0]$,

To that end we first verify that $Z_{0}^{\prime} \in \mathcal{Z}\left(r^{\prime}\right)$. This amounts to checking on a computer that

$$
\left|v_{0}\right|>|v|_{r^{\prime}} \quad \text { and } \quad 1-4 \frac{v_{0} \gamma_{0}}{\beta_{0}^{2}}>\left|F_{1}\right|_{r^{\prime}}+\left|F_{2}\right|_{r^{\prime}} \quad(\operatorname{cf.} \quad \text { (5.11) }) \text {. }
$$

Below we will use the following shorthand notation: $t^{\prime} \equiv\left|Z_{0}^{\prime}\right|_{r^{\prime}}$.

Set $z_{0}=0$, and define for all $n \geq 0$

$$
z_{n+1} \equiv N_{h}\left[z_{n}\right], \quad \Delta z_{n}=z_{n+1}-z_{n}, \quad Z_{n}^{\prime}=Z_{0}^{\prime}+M z_{n}
$$

(we have suppressed the dependence on $s$ in $Z_{n}^{\prime}$ for notational convenience), then

$$
\begin{aligned}
\left|\Delta z_{0}\right|_{r^{\prime}} & =\left|C_{h}\left[Z_{0}^{\prime}\right]-Z_{0}^{\prime}\right|_{r^{\prime}} \\
& \leq \frac{2 \delta \sqrt{2 c}}{c-4\left|a_{0}\right|\left|\Theta_{0}\right|_{\left(r^{\prime}, t^{\prime}\right)}-4\left|a_{0}\right| \delta} \quad(\mathrm{cf} . \text { equation (5.14) }),
\end{aligned}
$$

and

$$
\begin{aligned}
\left|\Delta z_{n}\right|_{r^{\prime}}= & \left|N_{h}\left[z_{n}\right]-N_{h}\left[z_{n-1}\right]\right|_{r^{\prime}} \\
= & \left|\Delta z_{n-1}+C_{h}\left[Z_{n-1}^{\prime}+M \Delta z_{n-1}\right]-C_{h}\left[Z_{n-1}^{\prime}\right]-M \Delta z_{n-1}\right|_{r^{\prime}} \\
\leq & \left|\left[C_{h}\left[Z_{n-1}^{\prime}+M \Delta z_{n-1}\right]-C_{h}\left[Z_{n-1}^{\prime}\right]-D C_{h}\left[Z_{n-1}^{\prime}\right] M \Delta z_{n-1}\right]\right|_{r^{\prime}}+ \\
& \left|\left[D C_{h}\left[Z_{n-1}^{\prime}\right]-D C_{h}\left[Z_{0}^{\prime}\right]\right] M \Delta z_{n-1}\right|_{r^{\prime}} \\
\equiv & T_{1}+T_{2} .
\end{aligned}
$$

To evaluate $T_{2}$ we will require estimates similar to (5.15)-(5.18). In fact, estimates (5.15)-(5.18) can be recycled after one substitutes $r^{\prime}$ for $r, \epsilon^{\prime}$ for $\epsilon$ and $s^{\prime} \equiv t^{\prime}+|M| \epsilon^{\prime}$ for $s$ (here $\epsilon^{\prime}$ is an a priori bound on $\left|z_{n}\right|_{r^{\prime}}, n \geq 0$, again, verifiable a posteriori). We will not repeat the details, but rather use the following symbolic notation

$$
\left|T_{2}\right|_{r^{\prime}} \leq C_{2}\left|\Delta z_{n-1}\right|_{r^{\prime}}
$$

keeping in mind that $C_{2}$ is computable on a machine.

To estimate $T_{1}$, we use a Cauchy bound for the following function:

$$
\begin{array}{r}
F(\zeta)=\frac{\sqrt{c-2 a_{0}\left[\Theta_{0}\left(\Pi_{1}, Z_{n-1}^{\prime}+\zeta M \Delta z_{n-1}\right)+h\left(P_{1}, Z_{n-1}^{\prime}+\zeta M \Delta z_{n-1}\right)+\right.}}{\left.\Theta_{0}\left(\Pi_{2}, Z_{n-1}^{\prime}+\zeta M \Delta z_{n-1}\right)+h\left(P_{2}, Z_{n-1}^{\prime}+\zeta M \Delta z_{n-1}\right)\right]},
\end{array}
$$


analytic on $\mathbb{D}_{R}$ with

$$
R \leq \frac{\rho-\left|Z_{n-1}^{\prime}\right|_{r^{\prime}}}{|M|\left|\Delta z_{n-1}\right|_{r^{\prime}}}
$$

This results in

$$
\begin{aligned}
\left|T_{1}\right|_{r^{\prime}} & \leq \frac{1}{R(R-1)} \frac{1}{2\left|a_{0}\right|} \sup _{|\zeta| \leq R}|F(\zeta)|_{r^{\prime}} \\
& =\frac{|M|^{2}\left|\Delta z_{n-1}\right|_{r^{\prime}}^{2}}{2\left|a_{0}\right|\left(\rho-s^{\prime}\right)\left(\rho-s^{\prime}-|M|\left|\Delta z_{n-1}\right|_{r^{\prime}}\right)} \sqrt{c+4\left|a_{0}\right|\left[\left|\Theta_{0}\right|_{\left(r^{\prime}, s^{\prime}\right)}+\delta\right]} \\
& \equiv C_{1} \frac{\left|\Delta z_{n-1}\right|_{r^{\prime}}^{2}}{\rho-s^{\prime}-|M|\left|\Delta z_{n-1}\right|_{r^{\prime}}}
\end{aligned}
$$

Finally, we have

$$
\begin{aligned}
\left|\Delta z_{n}\right|_{r^{\prime}} & \leq C_{1} \frac{\left|\Delta z_{n-1}\right|_{r^{\prime}}^{2}}{\rho-s^{\prime}-|M|\left|\Delta z_{n-1}\right|_{r^{\prime}}}+C_{2}\left|\Delta z_{n-1}\right|_{r^{\prime}} \\
& =K_{n-1}\left|\Delta z_{n-1}\right|_{r^{\prime}} \mid \\
K_{n-1} & \equiv C_{1} \frac{\left|\Delta z_{n-1}\right|_{r^{\prime}}}{\rho-s^{\prime}-|M|\left|\Delta z_{n-1}\right|_{r^{\prime}}}+C_{2}
\end{aligned}
$$

(we check that $K_{0}<1$ ).

Clearly, $K_{n}$ is a monotone increasing function of $\left|\Delta z_{n}\right|_{r^{\prime}}$, therefore $K_{n}<$ $K_{n-1}$. We can now verify that the sum

$$
\Sigma \equiv \sum_{k=0}^{\infty}\left|\Delta z_{k}\right|_{r^{\prime}}
$$

is convergent:

$$
\begin{aligned}
\Sigma & \leq \sum_{k=1}^{\infty} K_{k-1}\left|\Delta z_{k-1}\right|_{r^{\prime}}+\left|\Delta z_{0}\right|_{r^{\prime}} \\
& \leq \sum_{k=2}^{\infty} K_{k-1} K_{k-2}\left|\Delta z_{k-2}\right|_{r^{\prime}}+K_{0}\left|\Delta z_{0}\right|_{r^{\prime}}+\left|\Delta z_{0}\right|_{r^{\prime}} \\
& \leq \sum_{k=0}^{\infty} K_{0}^{k}\left|\Delta z_{0}\right|_{r^{\prime}} \\
& \leq \frac{\left|\Delta z_{0}\right|_{r^{\prime}}}{1-K_{0}}
\end{aligned}
$$

Therefore, for all $n \geq 0$

$$
\left|Z_{n}^{\prime}-Z_{0}^{\prime}\right|_{r^{\prime}} \leq|M| \frac{\left|\Delta z_{0}\right|_{r^{\prime}}}{1-K_{0}} \equiv \gamma^{\prime} .
$$

Finally, we verify that

$$
t^{\prime}+\gamma^{\prime}<\rho .
$$




\section{Acknowledgments}

The author is thankful to Hans Koch for many useful discussions on the subject.

\section{References}

IEEE, The IEEE Standard for Binary Floating-Point Arithmetic, ANSI/IEEE Std 754-1985.

J. J. Abad, H. Koch, Renormalization and periodic orbits for Hamiltonian flows, Comm. Math. Phys. 212 (2000) \# 2 371-394.

J. J. Abad, H. Koch and P. Wittwer, A renormalization group for Hamiltonians: numerical results, Nonlinearity 11 (1998) 1185-1194.

G. Benettin et al, Universal properties in conservative dynamical systems, Lettere al Nuovo Cimento 28 (1980) 1-4.

T. Bountis, Period doubling bifurcations and universality in conservative Systems, Physica 3D (1981) 577-589.

A. de Carvalho, M. Lyubich, M. Martens, Renormalization in the Hénon family, I: Universality but non-rigidity, J. Stat. Phys 121 (2005) 611-669.

P. Collet, J.-P. Eckmann and H. Koch, Period doubling bifurcations for families of maps on $\mathbb{R}^{n}$, J. Stat. Phys. 3D (1980).

P. Collet, J.-P. Eckmann and H. Koch, On universality for area-preserving maps of the plane, Physica 3D (1981) 457-467.

B. Derrida, Y. Pomeau, Feigenbaum's ratios of two dimensional area-preserving maps, Phys. Lett. A80 (1980) 217-219.

J.-P. Eckmann, H. Koch and P. Wittwer, Existence of a fixed point of the doubling transformation for area-preserving maps of the plane, Phys. Rev. A 26 (1982) \# 1 720-722.

J.-P. Eckmann, H. Koch and P. Wittwer, A Computer-Assisted Proof of Universality for Area-Preserving Maps, Memoirs of the American Mathematical Society 47 (1984), 1-121.

H. Epstein, New proofs of the existence of the Feigenbaum functions, Commun. Math. Phys. 106 (1986) 395-426.

H. Epstein, Fixed points of composition operators II, Nonlinearity 2 (1989) 305-310.

D. F. Escande, F. Doveil, Renormalization method for computing the threshold of the large scale stochastic instability in two degree of freedom Hamiltonian systems, J .Stat. Phys. 26 (1981) 257-284. 
E. de Faria, Proof of universality for critical circle mappings, Thesis, CUNY, 1992.

E. de Faria, Asymptotic rigidity of scaling ratios for critical circle mappings, Ergodic Theory Dynam. Systems 19 (1999), no. 4, 995-1035.

M. J. Feigenbaum, Quantitative universality for a class of nonlinear transformations, J. Stat. Phys. 19 (1978) 25-52.

M. J. Feigenbaum, Universal metric properties of non-linear transformations, $J$. Stat. Phys. 21 (1979) 669-706.

D. Gaidashev, Renormalization of isoenergetically degenerate Hamiltonian flows and associated bifurcations of invariant tori, Discrete Contin. Dyn. Syst. 13 (2005), no. 1, 63-102.

renormalization for Siegel disks and a constructive Measurable Riemann Mapping Theorem, Nonlinearity 20 (2007), no 3, 713-742.

D. Gaidashev, H. Koch, Renormalization and shearless invariant tori: numerical results, Nonlinearity 17 (2004), no. 5, 1713-1722.

D. Gaidashev, H. Koch, Period doubling in area-preserving maps: an associated one-dimenisonal problem, e-print math.DS/0811.2588 at Arxiv.org, to appear in Erg. Theor Dyn. Sys.

D. Gaidashev, M. Yampolsky, Cylinder renormalization of Siegel disks, Exp. Math. 16:2 (2007).

D. Gaidashev, T. Johnson, Dynamics of the Universal Area-Preserving Map Associated with Period Doubling: Hyperbolic Sets, Nonlinearity 22 2487-2520.

D. Gaidashev, T. Johnson, Dynamics of the Universal Area-Preserving Map Associated with Period Doubling: Stable Sets, J. Mod. Dyn. 3 (2009), no 4, $555-587$.

D. Gaidashev, T. Johnson, M. Martens, Rigidity for infinitely renormalizable area-preserving maps, preprint.

P. Hazard, M. Lyubich and M. Martens, Renormalizable Hénon-like maps and unbounded geometry, e-print math.DS/1002.3942 at Arxiv.org.

R. H. G. Helleman, Self-generated chaotic behavior in nonlinear mechanics, in "Fundamental problems in statistical mechanics", Ed. by E. G. D. Cohen, North-Holland, Amsterdam, p.165, (1980).

K. Khanin, J. Lopes Dias, J. Marklof, Multidimensional continued fractions, dynamic renormalization and KAM theory, Comm. Math. Phys., 270 (2007), no. $1,197-231$. 
H. Koch, On the renormalization of Hamiltonian flows, and critical invariant tori, Discrete Contin. Dyn. Syst. 8 (2002), 633-646.

H. Koch, A renormalization group fixed point associated with the breakup of golden invariant tori, Discrete Contin. Dyn. Syst. 11 (2004), no. 4, 881-909.

H. Koch, Existence of critical invariant tori, Ergodic theory and dynamical systems 28 1879-94

H. Koch, A. Schenkel and P. Wittwer, Computer-assisted proofs in analysis and programming in logic: a case study, SIAM Rev. 38 (1996), 565-604.

S. Kocić, Renormalization of Hamiltonians for Diophantine frequency vectors and KAM tori, Nonlinearity 18 (2005) 2513-2544.

O. E. Lanford, Computer-assisted proof of the Feigenbaum conjectures, Bull. A. M. S. (New Series) 6 (1982), 427-434.

O. E. Lanford, Computer-assisted proofs in analysis, Physica 124 A (1984), 465-470.

M. Lyubich, Feigenbaum-Coullet-Tresser universality and Milnor's hairness conjecture, Annals of Mathematics 149 (1999) 319-420.

M. Lyubich, M. Martens, Renormalization in the Hénon family, II: Homoclinic tangle, preprint, arXiv:mathDS/0804.0780 (2008).

R. S. MacKay, Renormalisation in area-preserving maps, Thesis, Princeton (1982). World Scientific, London (1993).

R. S. MacKay, Renormalization approach to invariant circles in area-preserving maps, Physica D7 (1983) 283-300.

A. Mehr and D.F. Escande, Destruction of KAM tori in Hamiltonian systems: link with the distabilization of nearby cycles and calculation of residues, Physica D13 (1984) 302-338.

C. McMullen, Self-similarity of Siegel disks and Hausdorff dimension of Julia sets, Acta Math. 180 (1998), 247-292.

S. J. Shenker, L. P. Kadanoff, Critical behaviour of KAM surfaces. I Empirical results, J. Stat. Phys. 27 (1982) 631-656.

C. Sparrow, The Lorenz equations : bifurcations, chaos, and strange attractors, New York ; Berlin : Springer (1982).

D. Sullivan, Bounds, quadratic differentials and renormalization conjectures, in: Mathematics into the Twenty-first Century, AMS Centennial Publications, Vol. II, Amer. Math. Soc., Providence, R.I. (1992) 417-466. 
C. Tresser and P. Coullet, Itérations d'endomorphismes et groupe de renormalisation, C. R. Acad. Sci. Paris 287A (1978), 577-580.

M. Yampolsky, Hyperbolicity of renormalization of critical circle maps, Publ. Math. Inst. Hautes Etudes Sci. 96 (2002), 1-41.

M. Yampolsky, Renormalization horseshoe for critical circle maps, Commun. Math. Physics 240 (2003), 75-96.

M. Yampolsky, Siegel disks and renormalization fixed points, Holomorphic Dynamics and Renormalization (Fields Inst. Commun. vol 53) (Providence, RI:AMS) pp. 377-93.

Programs available at http://math.uu.se/ gaidash 